\title{
Biological Characteristics and Molecular Mechanisms of Fludioxonil Resistance in Fusarium graminearum in China
}

\author{
F. Zhou, ${ }^{1,2}$ D. X. Li, ${ }^{1}$ H. Y. Hu, ${ }^{1}$ Y. L. Song, ${ }^{2}$ Y. C. Fan, ${ }^{2}$ Y. Y. Guan, ${ }^{1}$ P. W. Song, ${ }^{1}$ Q. C. Wei, ${ }^{1}$ H. F. Yan, ${ }^{3}$ and C. W. Li ${ }^{1, \dagger}$ \\ ${ }^{1}$ Henan Engineering Research Center of Crop Genome Editing, Henan Institute of Science and Technology, Xinxiang 453003, \\ China \\ ${ }^{2}$ Henan Engineering Research Center of Biological Fertilizer Developmental and Collaborative Application, Henan Institute of \\ Science and Technology, Xinxiang 453003, China \\ ${ }^{3}$ College of Plant Protection, Hebei Agricultural University, Baoding 071001, China
}

\begin{abstract}
Fusarium graminearum is the primary causal agent of Fusarium head blight (FHB) of wheat. The phenylpyrrole fungicide fludioxonil is not currently registered for the management of FHB in China. The current study assessed the fludioxonil sensitivity of a total of $53 \mathrm{~F}$. graminearum isolates collected from the six most important wheat-growing provinces of China during 2018 and 2019. The baseline fludioxonil sensitivity distribution indicated that all of the isolates were sensitive, exhibiting a unimodal cure with a mean effective concentration for $50 \%$ inhibition value of $0.13 \pm 0.12$ $\mu \mathrm{g} / \mathrm{ml}$ (standard deviation). Five fludioxonil-resistant mutants were subsequently induced by exposure to fludioxonil under laboratory conditions. Ten successive rounds of subculture in the absence of the selection pressure indicated that the mutation was stably inherited. However, the fludioxonilresistant mutants were found to have reduced pathogenicity, higher glycerol accumulation, and higher osmotic sensitivity than the parental wild-type iso-

as boscalid, carbendazim, tebuconazole, and fluazinam. Sequence analysis of four candidate target genes $(\mathrm{FgOs} 1, \mathrm{FgOs} 2, \mathrm{FgOs} 4$, and $\mathrm{FgOs} 5)$ revealed that the HBXT2R mutant contained two point mutations that resulted in amino acid changes at $\mathrm{K} 223 \mathrm{~T}$ and $\mathrm{K} 415 \mathrm{R}$ in its FgOs 1 protein, and one point mutation at residue 520 of its FgOs5 protein that resulted in a premature stop codon. Similarly, the three other mutants contained point mutations that resulted in changes at the K192R, K293R, and K411R residues of the $\mathrm{FgOs} 5$ protein but none in the $\mathrm{FgOs} 2$ and $\mathrm{FgOs} 4$ genes. However, it is important to point out that the $\mathrm{FgOs} 2$ and $\mathrm{FgOs} 4$ expression of all the fludioxonil-resistant mutants was significantly $(P<0.05)$ downregulated compared with the sensitive isolates (except for the SQ1-2 isolate). It was also found that one of the resistant mutants did not have changes in any of the sequenced target genes, indicating that an alternative mechanism could also lead to fludioxonil resistance.
\end{abstract} lates, indicating that there was a fitness cost associated with fludioxonil resistance. In addition, the study also found a positive cross resistance between fludioxonil, procymidone, and iprodione, but not with other fungicides such
Keywords: biological characteristics, cross resistance, fludioxonil, Fusarium graminearum, resistance mechanism
Fusarium head blight (FHB), which is predominantly caused by Fusarium graminearum (teleomorph Gibberella zeae), is the most important fungal disease affecting wheat production throughout the world (Haile et al. 2019; Qian et al. 2018; Rawat et al. 2016). FHB not only causes a substantial reduction in grain yield and quality but also results in the production of trichothecene mycotoxins and other secondary metabolites that pose a threat to both human and animal health (Blandino et al. 2012; Chilaka et al. 2017; Goswami and Kistler 2004; Qian et al. 2018; Qiu et al. 2018). Although the development of FHB-resistant wheat cultivars would be a management option (Al-Taweel et al. 2014), the breeding of such varieties has been hampered by a lack of resistant resources. Consequently, the management of FHB relies heavily on the application of chemical fungicides during wheat anthesis to ensure reliable crop production (Liu et al. 2019; Qian et al. 2018; Qiu et al. 2018; Willyerd et al. 2012). Commonly used fungicides include the benzimidazole fungicide carbendazim (Liu et al. 2019; Yuan and Zhou 2005; Zhang

${ }^{\dagger}$ Corresponding author: C. W. Li; lichengweiwau@ hotmail.com

Funding: This study was sponsored by the Key Scientific and Technological Research Project of Henan Province (numbers 192102110056 and 192102110131), and National Natural Science Foundation of China (number 31872129).

*The $\boldsymbol{e}$-Xtra logo stands for "electronic extra" and indicates that four supplementary tables are published online.

The author(s) declare no conflict of interest.

Accepted for publication 16 March 2020.

(C) 2020 The American Phytopathological Society et al. 2009), the cyanoacrylate fungicide phenamacril (Chen et al. 2011; Zhang et al. 2010), the demethylation inhibitors metconazole and tebuconazole (Qian et al. 2018; Tateishi et al. 2010), as well as the strobilurin azoxystrobin (Zhang et al. 2010). However, the extensive and repeated application of these chemicals has led to the frequent emergence of fungicide resistance that could jeopardize their effectiveness, as highlighted by incidences of carbendazim and tebuconazole resistance (Qian et al. 2018; Zhang et al. 2010).

The phenylpyrrole fungicide fludioxonil, which has broadspectrum activity against both basidiomycete and ascomycete pathogens, could provide a novel opportunity to control FHB (Brandhorst et al. 2019; Furukawa et al. 2012; Hu et al. 2019; Yoshimi et al. 2004). It is widely believed that the target site of fludioxonil is a histidine kinase (HK) related to the high-osmolarity glycerol (HOG1) stress response signal transduction pathway, although the precise mode of action has yet to be characterized fully (Brandhorst et al. 2019; Furukawa et al. 2012; Yoshimi et al. 2004). This signaling pathway is involved in many fungal processes, which perhaps explains why fludioxonil exhibits such a broad spectrum of activity against such a wide range of fungal pathogens (Brandhorst et al. 2019; Koch and Leadbeater 1992; Yoshimi et al. 2004). Indeed, fludioxonil has been used extensively against multiple plant pathogens in the past 30 years. In China, fludioxonil has been used control diseases of wheat, rice, cotton, and peanut for many years (Qiu et al. 2018; Sang et al. 2018). Although fludioxonil has not been widely applied in the field to control FHB, wheat seed are routinely treated with the fludioxonil containing formulation Beret Gold (Syngenta) (active ingredient: fludioxonil at $24.3 \mu \mathrm{g} / \mathrm{ml}$ ) before sowing to prevent soilborne diseases (Bailey et al. 2005; Qiu et al. 2018). Given such a history of heavy use, it is not surprising that fludioxonil-resistant isolates of $F$. graminearum have been detected in corn and soybean seedling samples (Broders et al. 2007), and a recent report has detailed the emergence of fludioxonil-resistant isolates of $F$. asiaticum 
in the wheat fields of China (Qiu et al. 2018). Although there have been no similar incidents of fludioxonil resistance in $F$. graminearum isolates in China, the emergence of resistance in the closely related species $F$. asiaticum has raised concerns that resistance could also arise in F. graminearum (Qiu et al. 2018). However, previous studies have shown that fludioxonil-resistant isolates often have reduced fitness and exhibit increased sensitivity to osmotic stress, which is consistent with observations that fludioxonil can inhibit the HOG1 cascade of the mitogen-activated protein (MAP) kinase signaling pathway (Lew 2010; Qiu et al. 2018; Sang et al. 2018). Indeed, studies in Botrytis cinerea have revealed that highly resistant laboratory mutants have altered sequences in the ATPase domain of the $\mathrm{C}$ terminal of an upstream HK, while field populations exhibit mutations predominantly distributed among the histidine kinases, adenyl cyclases, methyl accepting chemotaxis proteins, and phosphatases (HAMP) domains of the $\mathrm{N}$ terminal in $\mathrm{HK}$, a motif which is necessary for the recognition of stimulating compounds (Qiu et al. 2018; Ren et al. 2016; Sang et al. 2018). For example, mutations within the HAMP domains in the N-terminal region of the group III HK OS-1 in laboratory mutants have been shown to confer a high level of fludioxonil resistance (Duan et al. 2014a; Fillinger et al. 2012). In addition, mutant $O s 1$ alleles in Neurospora crassa have been reported to have either single or multiple mutations within the six-tandem-repeat motif (Miller et al. 2002), while studies on mutant strains of Cochliobolus heterostrophus and $N$. crassa have also indicated that resistance to dicarboximide and phenylpyrrole fungicides is influenced by the structure of the amino acid repeat region (Yoshimi et al. 2004). However, some plant pathogens are known to have multiple $H K$ genes, indicating that mutations in other $O s$-like genes may be possible causes of fludioxonil resistance (Catlett et al. 2003; Qiu et al. 2018).

Although fludioxonil resistance has been reported in field isolates of $F$. asiaticum (Qiu et al. 2018), and low levels of resistance have been detected in many plant pathogens (Avenot and Michailides 2015; Dry et al. 2004; Duan et al. 2014a; Han et al. 2017; Jung et al. 2012; Li and Xiao 2008; Peters et al. 2008; Ren et al. 2016; Tückmantel et al. 2011; Wu et al. 2015), the precise mechanism of fludioxonil resistance has yet to be characterized in detail. Further research is required to elucidate the biological characteristics and molecular mechanism of fludioxonil resistance. The specific objectives of the current study were to (i) establish the baseline sensitivity of $F$. graminearum to fludioxonil; (ii) compare the fitness parameters and physiological characteristics of sensitive and fludioxonil-resistant isolates; (iii) determine whether there are any patterns of cross resistance between fludioxonil and other widely used fungicides, including procymidone, iprodione, boscalid, carbendazim, tebuconazole, and fluazinam; and (iv) identify potential mechanism of fludioxonil resistance in $F$. graminearum.

\section{Materials and Methods}

Isolates of $\boldsymbol{F}$. graminearum, media, and mycelia preparation. Infected wheat samples were collected from six mainly wheatgrowing provinces of China (Henan, Heibei, Anhui, Jiangsu, Sichuan, and Guizhou Provinces) in summer 2018 and 2019 (Supplementary Table S1). The samples were collected from wheat grains exhibiting typical symptoms of FHB, and individual $F$. graminearum isolates were purified by single-spore isolation according to the protocol of a previous study (Qiu et al. 2018). The fungal cultures used in the study were routinely maintained on potato dextrose agar (PDA; potato at $200.0 \mathrm{~g} / \mathrm{liter}$, agar at $20.0 \mathrm{~g} / \mathrm{liter}$, and dextrose at $20.0 \mathrm{~g} /$ liter), and their spores suspended in a $20 \%$ sterile-glycerol solution for long-term storage at $-20^{\circ} \mathrm{C}$.

Five fludioxonil-sensitive isolates, including 2XZ-4, HBXT2, CM2, SQ1-2, and YN1-3, which had effective concentration for 50\% inhibition $\left(\mathrm{EC}_{50}\right)$ values of $0.06,0.01,0.05$, and $0.04 \mu \mathrm{g} / \mathrm{ml}$, respectively, were selected for further study. Repeated fludioxonil exposure resulted in the selection of the five highly resistant laboratory mutants $2 X Z-4 R$, HBXT2R, CM2R, SQ1-2R, and YN1-3R with $\mathrm{EC}_{50}$ values of 41.36, $48.75,68.45,101.78$, and $73.21 \mu \mathrm{g} / \mathrm{ml}$, respectively.

The cultures used for the intracellular glycerol accumulation assay and genomic DNA extraction were prepared in a conical flask according to the protocols of previous studies (Qiu and Shi 2014; Qiu et al. 2018). Each isolate was incubated in potato dextrose broth (PDB) medium (Beijing Aoboxing Bio-Tech Co. Ltd.) for $72 \mathrm{~h}$ at $23^{\circ} \mathrm{C}$ with shaking $(130 \mathrm{rpm})$. The mycelium was then harvested and washed three times in PDB before being flash-frozen in liquid nitrogen. In the case of the glycerol assay, an additional step involving the transfer of the harvested mycelium to fresh PDB amended with $0.1 \mu \mathrm{M}$ fludioxonil and incubation at $23^{\circ} \mathrm{C}$ for a further $5 \mathrm{~h}$ was included, before the mycelium was harvested a second time, washed, and flash-frozen in liquid nitrogen.

Fungicides. Technical-grade fungicides used in the study, including 95.3\% iprodione (Heyi Agrochemical Co. Ltd.), 98.0\% procymidone (Heyi Agrochemical Co. Ltd.), 97.0\% boscalid (Zhejiang Heben Pesticide \& Chemicals Co. Ltd.), 96.2\% tebuconazole (Sheyang Huanghai Pesticide Chemical Co. Ltd.), and 96.0\% fluazinam (Hubei Jianyuan Chemical Co. Ltd.), were dissolved in acetone to produce stock solutions of $10,000 \mu \mathrm{g} / \mathrm{ml}$, while the $98.1 \%$ carbendazim (Haili Guixi Chemical Co. Ltd.) was dissolved in hydrochloric acid at $0.1 \mathrm{~mol} /$ liter (Supplementary Table S2). The stock solutions were stored at $4^{\circ} \mathrm{C}$ for no longer than 2 weeks before being used to prepare the serial dilutions used in the experiments. Mycelial growth assays were used to confirm that the solvents had no effect on the growth of $F$. graminearum at the range of concentrations tested (data not shown).

Baseline sensitivity of $\boldsymbol{F}$. graminearum to fludioxonil. In total, $53 \mathrm{~F}$. graminearum isolates were used to determine baseline $\mathrm{EC}_{50}$ values for fludioxonil using the mycelial growth assay described in a previous study (Zhou et al. 2017). Briefly, fresh mycelium plugs (5 $\mathrm{mm}$ in diameter) from 3-day-old cultures were transferred to PDA containing fludioxonil at $0,0.001,0.005,0.025,0.125$, $0.625,3.125$, and $15.625 \mu \mathrm{g} / \mathrm{ml}$. The radial growth of the resulting mycelium was measured after $72 \mathrm{~h}$ of incubation at $23^{\circ} \mathrm{C}$. The percent inhibition was then calculated using the following formula: 1 - (radial growth on fungicide-amended PDA medium/radial growth on PDA medium) $\times 100$, while the $\mathrm{EC}_{50}$ values were estimated by linear regression of $\log _{10}$ fungicide concentrations versus the probit value of percent growth inhibition. Each isolate was represented by three separate plates and the entire experiment was performed twice.

Heritable stability of fludioxonil resistance. The hereditary stability of the five fludioxonil-resistant $F$. graminearum mutants ( $2 \mathrm{XZ}$ 4R, HBXT2R, CM2R, SQ1-2R, and YN1-3R) was assessed with a modified version of the protocol used by $\mathrm{Hu}$ et al. (2019). Each mutant was subjected to 10 successive rounds of subculture on fludioxonil-free PDA before their fludioxonil $\mathrm{EC}_{50}$ values were reevaluated at the following concentrations: $0,1,5,50,100,200$, 300 , and $600 \mu \mathrm{g} / \mathrm{ml}$. The resistance factor (RF) was then calculated by comparing the $\mathrm{EC}_{50}$ values of the resistant mutants to those of their parental isolates according to the following formula: $\mathrm{RF}=$ $\mathrm{EC}_{50}$ of the mutant/EC $\mathrm{E}_{50}$ of parent isolate. The stability of resistance itself was estimated from the change in $\mathrm{EC}_{50}$ values calculated for the 1 st and 10th subculture. Each isolate was represented by three replicate plates and the entire experiment was performed twice.

Biological characteristics of fludioxonil-resistant $F$. graminearum mutants. Mycelial growth. The mycelial growth of four sensitive wild-type $F$. graminearum isolates (2XZ-4, HBXT2, $\mathrm{CM}$ 2, and SQ1-2) and five laboratory fludioxonil-resistant mutants (2XZ-4R, HBXT2R, CM2R, SQ1-2R, and YN1-3R) was evaluated as previously described (Zhou et al., 2020). Briefly, mycelial plugs ( $5 \mathrm{~mm}$ in diameter) were taken from the edge of 2-day-old colonies and transferred to fresh PDA plates that were then incubated at $23^{\circ} \mathrm{C}$ with a 12-h photoperiod. The resulting colonies were observed daily and the diameter of each measured at 24,48 , and $72 \mathrm{~h}$ postinoculation. Each isolate was represented by six separate plates and the entire experiment was performed once.

Sporulation. The rate of sporulation of the sensitive and resistant isolates was assessed on PDA using the method of Duan et al. (2018), with a few modifications. The test colonies were initially established by transferring 5-mm mycelial plugs from 2-day-old PDA cultures to flasks containing $30 \mathrm{ml}$ of mung bean broth. After 
3 days of incubation at $23^{\circ} \mathrm{C}$ with shaking $(130 \mathrm{rpm})$, the resulting spores were harvested and counted using a hemocytometer (Shanghai Qiujing Biochemical Reagent Instrument Co., Ltd.). Each isolate was represented by at least three replicate flasks, and the entire experiment was performed twice.

Sensitivity to osmotic pressure. The response of the resistant and sensitive isolates to osmotic stress was performed according to the protocol of a previous study (Qiu et al. 2018), with a few modifications. Mycelial plugs $(5 \mathrm{~mm})$ from 2-day-old PDA cultures were transferred to fresh PDA plates amended with $0.5 \mathrm{M} \mathrm{KCl}, 0.5 \mathrm{M}$ glucose, $0.5 \mathrm{M} \mathrm{MgCl}_{2}$, or $0.5 \mathrm{M}$ mannitol. Identical amendment-free cultures were used as the control. The diameters of the colonies were measured after $48 \mathrm{~h}$ of incubation at $23^{\circ} \mathrm{C}$ with a 12 -h photoperiod, and the percent inhibition of mycelial growth was calculated according to the formula detailed in the previous study (Qiu et al. 2018). Each treatment was represented by six replicate plates, and the entire experiment performed twice.

Pathogenicity on wheat. The pathogenicity of the resistant and sensitive isolates was assessed on wheat seedlings using the in vitro assay described in previous studies (Duan et al. 2018; Liu et al. 2013; Yang et al. 2018; Zhang et al. 2017), with a few minor modifications. Wheat coleoptiles from 3-day-old seedlings (cultivar Bainong 207) were cut, and their exposed surfaces were inoculated with $2-\mu 1$ spore suspensions $\left(10^{5}\right.$ spores $\left./ \mathrm{ml}\right)$, or water in the case of the negative controls. The seedlings were then maintained at $23^{\circ} \mathrm{C}$ with $95 \%$ relative humidity and a 16-h photoperiod, and the length of the infection lesions was determined at 15 days postinoculation. Each isolate was represented by at least 15 replicate coleoptiles, and the entire experiment was performed twice.

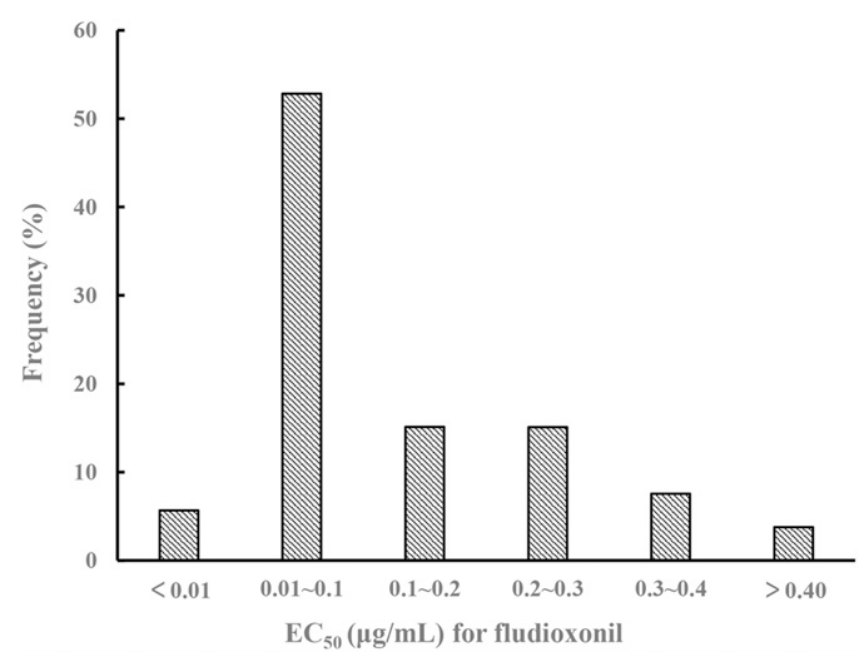

Fig. 1. Frequency distribution of fludioxonil effective concentration for $50 \%$ inhibition $\left(\mathrm{EC}_{50}\right)$ values for 53 Fusarium graminearum isolates collected from the six primary wheat-producing provinces of China during the summers of 2018 and 2019.

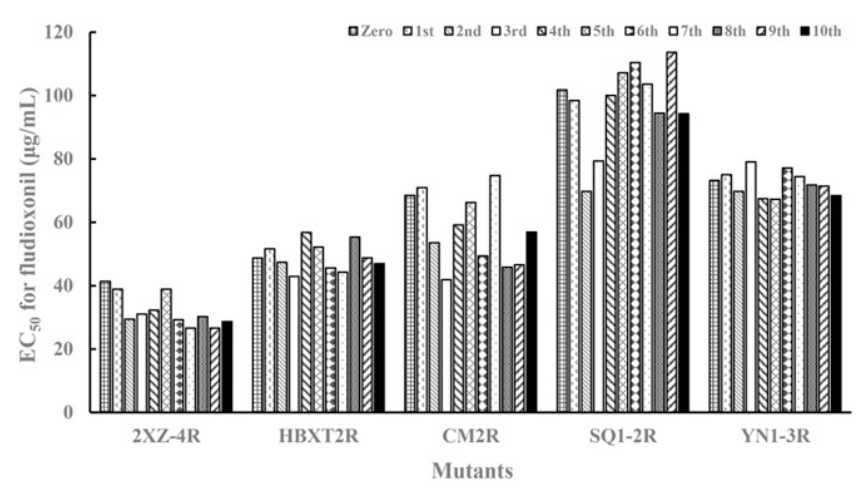

Fig. 2. Heritable stability of fludioxonil resistance in five Fusarium graminearum mutants assessed by the variation in fludioxonil effective concentration for $50 \%$ inhibition $\left(E C_{50}\right)$ values during 10 successive rounds of subculture.
Determination of glycerol content. The glycerol accumulation of the resistant and sensitive isolates was determined using the protocol applied in two previous studies (Zhang et al. 2017; Zhou et al. in press), with a few modifications. $F$. graminearum cultures were first established in $75 \mathrm{ml}$ of PDB media by inoculation with a single mycelial plug $(5 \mathrm{~mm})$. The test cultures were then incubated for 3 days at $23^{\circ} \mathrm{C}$ with shaking $(150 \mathrm{rpm})$ before the mycelium from each was harvested, washed three times in PDB, and transferred to fresh media in the absence or presence of fludioxonil at $0.1 \mu \mathrm{g} / \mathrm{ml}$. After a further $5 \mathrm{~h}$ of incubation, the mycelia were reharvested, washed, and ground under liquid nitrogen using a grinder, before being transferred to the glycerol extraction buffer. The glycerol content was then measured using a commercial glycerol assay kit (Applygen, Beijing, China) according to the instructions of the manufacturer. Each isolate was represented by at least three replicate samples, and the entire experiment was performed twice.

Cloning and sequencing of the $\mathrm{FgOs} 1, \mathrm{FgOs}-2, \mathrm{FgOs} 4$, and FgOs5 genes. Fresh mycelia were collected from 200-ml PDB cultures, and their genomic DNA was extracted according to the protocol of De Miccolis Angelini et al. (2014). Primer sets developed in a previous study (Qiu et al. 2018) were then used to amplify the full-length sequence of each candidate gene. The primers had the following sequences: FgOs1F1/FgOs1R1, ACCCACCCGTTCAAACTACAC/ ATCTCGCCTGATGCCTCTAC; FgOs1F2/FgOs1R2, CAAGCCT GAACACGAACAAC/AGCAACGAATAACCAGAGCC; FgOs2F/ FgOs2F, ACCACACCTATCAAACCACTGC/TTCCCTTATCTCCC CAACG; FgOs4F1/FgOs4R1, GCAGCCACAGCAAGACGAA/CGG GGACGCAATCACATAGA; FgOs4F2/FgOs4R2, CGACTGAAAT GAGCAAACGC/AGAAGAAAGAGGAAGTGAAAG; and FgOs5F/ FgOs5R, TTACCGTCCCTGGGATTCTAC/CCTGCCTTCCTTATC TTGTCTT. The PCRs were performed using 50- $\mu 1$ reaction mixtures containing $25 \mu \mathrm{l}$ of $2 \times$ ES Taq Master Mix, $1.5 \mu$ l of template DNA, $2 \mu \mathrm{l}$ of each primer, and $21.5 \mu \mathrm{l}$ of double-distilled $\mathrm{H}_{2} \mathrm{O}$ according to the PCR kit obtained from CoWin Biosciences, and processed using a 96-well thermal cycler (Applied Biosystems, Thermo Fisher Scientific) with the following program: an initial denaturation at $94^{\circ} \mathrm{C}$ for $2 \mathrm{~min}$; followed by 35 cycles of $94^{\circ} \mathrm{C}$ for $30 \mathrm{~s}, 57^{\circ} \mathrm{C}$ for $30 \mathrm{~s}$, and $72^{\circ} \mathrm{C}$ for $2.5 \mathrm{~min}$; and a final extension at $72^{\circ} \mathrm{C}$ for $10 \mathrm{~min}$. The resulting PCR products were then purified and cloned into the PMD19-T vector using a cloning kit (TaKaRa) and sequenced commercially (Wuhan Genecreate Biotechnology Co. Ltd). The predicted amino acid sequences were then aligned using the DNAMAN software package (ver.8.0; Lynnon Biosoft), and amino acid differences between resistant and sensitive isolates were determined as described previously (Gong et al. 2018; Zhou et al. 2017, 2020).

Comparison of expression levels of $\mathrm{FgOs} 2$ and $\mathrm{FgOs}_{4}$ genes in fludioxonil-resistant mutants of $\boldsymbol{F}$. graminearum. Total RNA was extracted from mycelia using a fungal RNA kit (Omega Bio-Tek) according to the manufacturer's instructions, and used as a template for the amplification of $\mathrm{FgOs} 2$ and $\mathrm{FgOs} 4$ genes (about $400 \mathrm{bp}$ ) using the following primer pairs: FgOs2 qPCR F1/FgOs2 qPCR R1, FgOs4 qPCR F1/FgOs4 qPCR R1, and Fg $\beta$-tubulin F1/Fg $\beta$-tubulin $\mathrm{F} 1$, which had the sequences CATGCGTGGACTCAAGTACG/ GAGCTCGGTGATGATGGAGA, ACAAAGCTTGCCCTTGACTG/ CCTCGTCGTTCATCATTCGG, and GCGAGGTTGAGAACTGTG AC/GCGAGGTTGAGAACTGTGAC, respectively. First-strand cDNA was prepared with the PrimeScript RT reagent kit (TaKaRa). Real-time PCR amplifications were performed on applied biosystems of QuantStudio 6 Flex PCR detection systems (Thermo Fisher) using SYBR Green I fluorescent dye detection. The relative quantities were calculated using the described methods previously (Duan et al. 2018). The $\beta$-tubulin as a reference gene was used in this study, and three biological replicates for each mutant were used to calculate the mean and standard error.

Cross resistance between fludioxonil and other fungicides. The potential cross resistance between fludioxonil and six commonly used fungicides, including carbendazim, iprodione, procymidone, boscalid, tebuconazole, and fluazinam, was assessed using the mycelial growth assay approach, as described previously (Zhou et al. 2014 , in press). The same protocol used to determine the fludioxonil $\mathrm{EC}_{50}$ values described above was used to determine similar values 
for each of the different fungicides in each of the fludioxonil-resistant and -sensitive isolates. At least seven concentrations were used for each fungicide: $0,1.5625,3.125,6.25,12.5,25,50$, and $100 \mu \mathrm{g} /$ $\mathrm{ml}$ in the case of fludioxonil, iprodione, and procymidone; and 0 , $0.0625,0.0125,0.025,0.05,0.10,0.20,0.40,0.80$, and $1.60 \mu \mathrm{g} / \mathrm{ml}$ with carbendazim, boscalid, fluazinam, and tebuconazole. Each treatment was represented by at least three replicate plates, and the entire experiment was conducted twice.

Data analysis. The data collected during the current study were first evaluated by analysis of variance using SPSS software (ver. 17.0; SPSS Inc.). Fisher's least significant difference test $(\alpha=$ 0.05 and $\alpha=0.01$ ) was then used to determine the statistical differences between different treatments. The DNA sequencing data were analyzed using the software DNAMAN (version 6.0; Lynnon Biosoft).

\section{Results}

Baseline sensitivity of $\boldsymbol{F}$. graminearum to fludioxonil. The current study found that none of the $53 \mathrm{~F}$. graminearum isolates collected from wheat-growing provinces of China were able to grow on PDA amended with fludioxonil at $5 \mathrm{mg} / \mathrm{ml}$. The frequency distribution of their $\mathrm{EC}_{50}$ values conformed to a unimodal distribution (Fig. 1), with values ranging from 0.01 to $0.45 \mu \mathrm{g} / \mathrm{ml}$ and a mean of $0.13 \pm 0.12 \mu \mathrm{g} / \mathrm{ml}$ ( \pm standard deviation [SD]). All 53 wild-type isolates were completely inhibited by a fludioxonil concentration of $5.0 \mu \mathrm{g} / \mathrm{ml}$.

Hereditable stability, biological characteristics, and osmotic sensitivity associated with fludioxonil resistance. After 10 successive rounds of subculturing, the $\mathrm{EC}_{50}$ values of the five fludioxonilresistant mutants had not changed significantly (Fig. 2; Supplementary

Table 1. Mycelial growth, sporulation, and pathogenicity of five fludioxonil-resistant mutants and four sensitive isolates of Fusarium graminearum ${ }^{\mathrm{v}}$

\begin{tabular}{|c|c|c|c|c|c|}
\hline \multirow[b]{2}{*}{ Isolate $\left(\mathbf{E C}_{50}\right)^{\mathbf{w}}$} & \multicolumn{3}{|c|}{ Mycelial growth $(\mathbf{c m}) \pm \mathrm{SE}^{\mathrm{x}}$} & \multirow[b]{2}{*}{ Spores/mly } & \multirow[b]{2}{*}{ Pathogenicity $^{z}$} \\
\hline & $24 \mathrm{~h}$ & $48 \mathrm{~h}$ & $72 \mathrm{~h}$ & & \\
\hline $2 X Z-4(0.06)$ & $1.18 \pm 0.05 \mathrm{a} \mathrm{A}$ & $3.13 \pm 0.15 \mathrm{a} \mathrm{A}$ & $5.73 \pm 0.03 \mathrm{c} \mathrm{B}$ & $10.17 \pm 1.19$ a A & $2.63 \pm 0.07 \mathrm{~d} \mathrm{DEF}$ \\
\hline HBXT2 (0.01) & $1.75 \pm 0.07 \mathrm{bc} \mathrm{BC}$ & $3.23 \pm 0.05$ a A & $4.78 \pm 0.21 \mathrm{a} \mathrm{A}$ & $9.33 \pm 0.84$ a A & $2.37 \pm 0.36 \mathrm{~cd} \mathrm{DEF}$ \\
\hline CM2 (0.05) & $2.05 \pm 0.10 \mathrm{~d} \mathrm{C}$ & $4.55 \pm 0.07 \mathrm{c} \mathrm{C}$ & $5.70 \pm 0.00 \mathrm{bc} \mathrm{B}$ & $25.34 \pm 0.38 \mathrm{bc} \mathrm{AB}$ & $3.50 \pm 0.26$ e F \\
\hline SQ1-2 (0.04) & $1.90 \pm 0.10 \mathrm{~cd} \mathrm{BC}$ & $4.23 \pm 0.09 \mathrm{bc} \mathrm{BC}$ & $5.70 \pm 0.00 \mathrm{bc} \mathrm{B}$ & $20.50 \pm 3.71 \mathrm{ab} \mathrm{AB}$ & $2.73 \pm 0.15$ de $\mathrm{EF}$ \\
\hline 2XZ-4R (41.36) & $1.60 \pm 0.10 \mathrm{bc} \mathrm{B}$ & $3.98 \pm 0.08 \mathrm{~b} \mathrm{~B}$ & $5.55 \pm 0.05 \mathrm{bc} \mathrm{B}$ & $20.67 \pm 1.80 \mathrm{ab} \mathrm{AB}$ & $0.00 \pm 0.00 \mathrm{a} \mathrm{A}$ \\
\hline HBXT2R (48.75) & $1.50 \pm 0.04 \mathrm{~b} \mathrm{AB}$ & $3.35 \pm 0.19$ a A & $5.48 \pm 0.17 \mathrm{~b} \mathrm{~B}$ & $38.33 \pm 6.29 \mathrm{~cd} \mathrm{BC}$ & $1.67 \pm 0.20 \mathrm{bc} \mathrm{CDE}$ \\
\hline CM2R (68.45) & $1.65 \pm 0.10 \mathrm{bc} B C$ & $4.15 \pm 0.16 \mathrm{~b} \mathrm{BC}$ & $5.58 \pm 0.05 \mathrm{bc} \mathrm{B}$ & $40.17 \pm 8.09 \mathrm{~d} \mathrm{C}$ & $1.50 \pm 0.29 b$ BCD \\
\hline SQ1-2R (101.78) & $1.78 \pm 0.16$ bcd BC & $4.25 \pm 0.07 \mathrm{bc} \mathrm{BC}$ & $5.70 \pm 0.00 \mathrm{bc} \mathrm{B}$ & $34.17 \pm 4.30 \mathrm{bc} \mathrm{BC}$ & $0.83 \pm 0.15 a b$ ABC \\
\hline YN1-3R (73.21) & $1.88 \pm 0.11 \mathrm{~cd} \mathrm{BC}$ & $3.98 \pm 0.10 \mathrm{~b} \mathrm{~B}$ & $5.73 \pm 0.03$ c B & $20.33 \pm 2.23 \mathrm{ab} \mathrm{AB}$ & $0.47 \pm 0.15 \mathrm{a} \mathrm{AB}$ \\
\hline
\end{tabular}
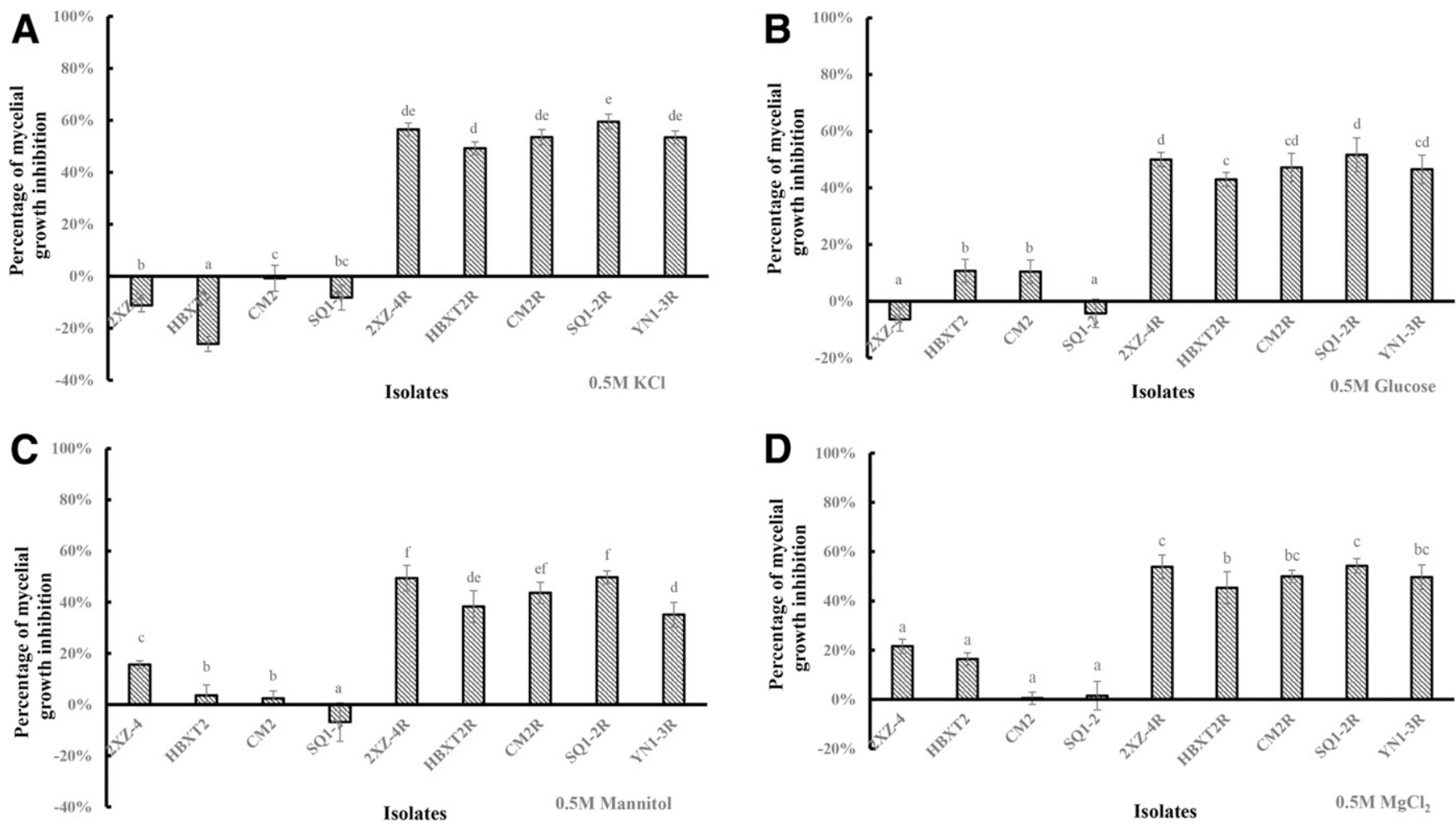

Fig. 3. Mycelial growth of five fludioxonil-resistant mutants and four sensitive isolates of Fusarium graminearum in response to osmotic stress induced by $\mathbf{A}, 0.5 \mathrm{M} \mathrm{KCl} ; \mathbf{B}, 0.5 \mathrm{M}$ glucose; C, $0.5 \mathrm{M}$ mannitol; and $\mathbf{D}, 0.5 \mathrm{M} \mathrm{MgCl}_{2}$. Error bars indicate the standard error from two separate experiments. Different letters above columns indicate significant differences according to Fisher's least significant difference test $(\alpha=0.05)$. 
Table S3), indicating that the resistance was stable. Investigation of their biological characteristics indicated that the mycelial growth of the fludioxonil-resistant mutants was little affected but that the sporulation of four mutants (HBXT2R, CM2R, SQ1-2R, and YN1-3R) was significantly increased relative to the sensitive wild-type isolates (Table 1). However, the pathogenicity of all the fludioxonil-resistant isolates was significantly reduced $(P<0.05)$, with the mutant $2 \mathrm{XZ}-4 \mathrm{R}$ completely losing its ability to infect wheat coleoptiles (Table 1). In addition, it was also found that all of the fludioxonil-resistant mutants had significantly $(P<0.05)$ reduced growth rates in response to osmotic stress as assessed on PDA amended with $0.5 \mathrm{M} \mathrm{KCl}$ (Fig. 3A), $0.5 \mathrm{M}$ glucose (Fig. 3B), $0.5 \mathrm{M}$ mannitol (Fig. 3C), and $0.5 \mathrm{M} \mathrm{MgCl}_{2}$ (Fig. 3D). Taken together, these results indicate that the fludioxonilresistant mutants had significantly reduced fitness with regard to both pathogenicity and osmotic stress.

Glycerol accumulation in fludioxonil-resistant mutants of F. graminearum. The glycerol content of all the fludioxonil-resistant mutants was significantly increased relative to the sensitive wild-type isolates when grown on PDB in the absence of fludioxonil (Fig. 4). However, with the addition of fludioxonil at $0.1 \mu \mathrm{g} / \mathrm{ml}$, this trend was reversed, with the sensitive isolates 2XZ-4, HBXT2, CM2, and SQ1-2 generally having a higher glycerol content, increased by 3.35-, 1.79-, 1.96-, and 2.09-fold, respectively. In contrast, the fludioxonil treatment had a much less dramatic, although still significant, effect on the fludioxonil-resistant mutants, increasing by just 1.30-, 1.09-, 1.06-, 1.27-, and 1.07-fold for isolates 2XZ-4R, HBXT2R, CM2R, SQ1-2R, and YN1-3R, respectively (Fig. 4).

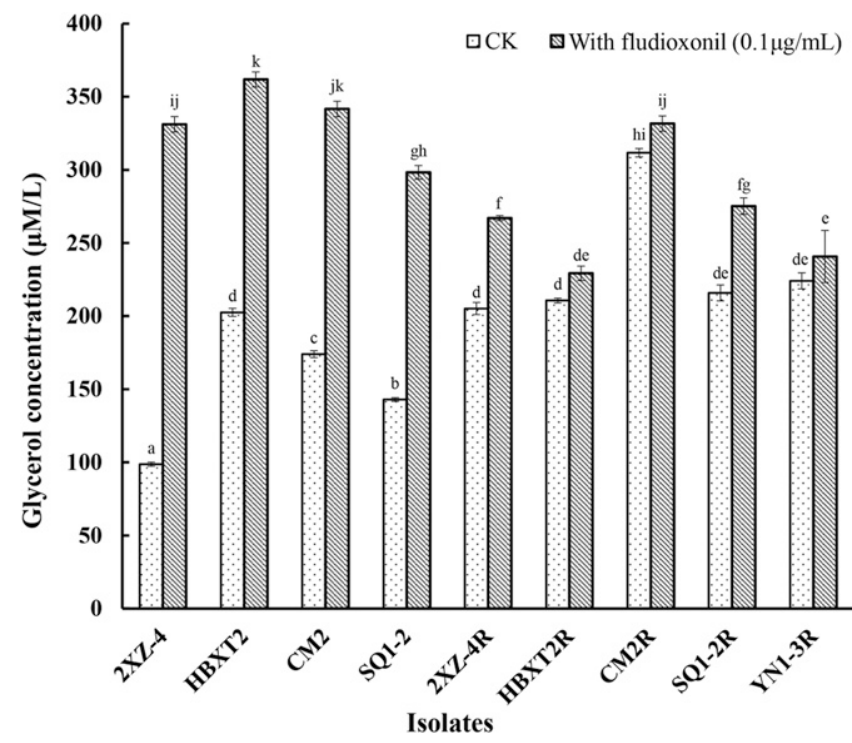

Fig. 4. Glycerol content of five resistant mutants and four sensitive isolates of Fusarium graminearum in response to fludioxonil treatment. Error bars indicate the standard error from two separate experiments. Different letters above columns indicate significant differences according to Fisher's least significant difference test $(\alpha=0.05)$.
Cross resistance with other fungicides in fludioxonil-resistant mutants of $\boldsymbol{F}$. graminearum. The current study found a positive cross resistance between fludioxonil and the dicarboximide fungicides procymidone and iprodione. This is not surprising because the dicarboximides are known to share similar modes of action with fludioxonil and also target the HOG-MAP kinase signal transduction pathway. However, no cross resistance was found between fludioxonil and any of the other fungicides tested, including carbendazim, boscalid, tebuconazole, and fluazinam (Table 2).

Sequence analysis of the $\mathrm{FgOs} 1, \mathrm{FgOs} 2, \mathrm{FgOs} 4$, and $\mathrm{FgOs} 5$ genes in fludioxonil-resistant mutants of $F$. graminearum. The open reading frame sequences of the four $F$. graminearum $O s$ genes ( $F g O s 1, F g O s 2, F g O s 4$, and $\mathrm{FgOs} 5$ ), which have previously been associated with fludioxonil resistance in $F$. asiaticum, were successfully cloned from the five fludioxonil-resistant mutants and four wild-type isolates. The predicted protein sequences identified several amino acid changes (Supplementary Table S4). HBXT2R had two point mutations, K223T and $\mathrm{K} 415 \mathrm{R}$, in its $\mathrm{FgOs} 1$ gene, as well as one in its $\mathrm{FgOs} 5$ gene that resulted in a premature stop codon at amino acid 520. Three of the other mutants, 2XZ-4R, CM2R, and SQ1-2R, had single mutations in their $\mathrm{FgOs} 5$ gene, which resulted in the amino acid changes of K192R, K293R, and K411R, respectively. However, it was interesting to note that the fifth mutant, YN1-3R, had no mutations in any of the sequenced target genes, indicating that an alternative resistant mechanism was responsible for its reduced sensitivity to fludioxonil (Table 3). No amino acid changes were detected in the predicted $\mathrm{FgOs} 2$ and $\mathrm{FgOs} 4$ sequences of any of the mutants tested.

Comparison of expression levels of $\mathrm{FgOs} 2$ and $\mathrm{FgOs}_{4}$ genes in fludioxonil-resistant mutants of $F$. graminearum. In this study, the expression levels of $\mathrm{FgOs} 2$ and $\mathrm{FgOs} 4$ in all isolates were assessed. Results showed that $\mathrm{FgOs} 2$ and $\mathrm{FgOs} 4$ expressions of all the fludioxonil-resistant mutants significantly $(P<0.05)$ downregulated compared with the sensitive isolates (except for the SQ1-2 isolates) when grown in PDB media. Meanwhile, similar results were also observed when the isolates were treated with fludioxonil at $0.1 \mu \mathrm{g} / \mathrm{ml}$ (Fig. 5). These results indicated that the candidate target genes $\mathrm{FgOs} 2$ and $\mathrm{FgOs} 4$ maybe also related to the fludioxonil resistance.

\section{Discussion}

The FHB caused by $F$. graminearum is an important fungal disease affecting wheat production throughout the world (Haile et al. 2019; Qian et al. 2018; Rawat et al. 2016). Previous research has shown that several fungicides, including carbendazim, tebuconazole and phenamacril, are at high risk for the development of resistance, jeopardizing their capacity to control FHB in China (Chen et al. 2011; Duan et al. 2014b; Liu et al. 2019; Qian et al. 2018). However, inappropriate use or overuse can lead to an increased selection pressure on pathogens that results in fungicide resistance (Brent and Hollomon 1998). The current study evaluated $53 \mathrm{~F}$. graminearum isolates collected from the six primary wheat-producing provinces of China in summer 2018 and 2019, and found that all of these wild-type isolates remained sensitive to fludioxonil, having a baseline fludioxonil sensitivity with a mean $\mathrm{EC}_{50}$ value of $0.13 \pm$ $0.12 \mu \mathrm{g} / \mathrm{ml}$ (SD). Further investigation of five fludioxonil-resistant mutants produced by exposure to fludioxonil under laboratory

Table 2. Cross resistance between fludioxonil and six commonly used fungicides in Fusarium graminearum $^{\mathrm{z}}$

\begin{tabular}{|c|c|c|c|c|c|c|c|c|c|}
\hline \multirow[b]{2}{*}{ Fungicides } & \multicolumn{4}{|c|}{ Sensitive isolates } & \multicolumn{5}{|c|}{ Fludioxonil-resistant mutants } \\
\hline & $2 X Z-4$ & HBXT2 & CM2 & SQ1-2 & 2XZ-4R & HBXT2R & CM2R & SQ1-2R & YN1-3R \\
\hline Fludioxonil & $0.07^{\mathrm{z}}$ & 0.07 & 0.03 & 0.03 & 41.36 & 48.75 & 68.45 & 101.78 & 73.21 \\
\hline Procymidone & 0.35 & 0.46 & 0.42 & 0.77 & 36.88 & $>50.00$ & $>50.00$ & 78.65 & $>50.00$ \\
\hline Iprodione & 0.87 & 1.77 & 0.72 & 1.36 & 0.99 & 96.78 & 68.96 & $>50.00$ & $>50.00$ \\
\hline Boscalid & 0.10 & 0.14 & 0.08 & 0.01 & 0.16 & 0.22 & 0.07 & 0.31 & 0.26 \\
\hline Carbendazim & 0.730 & 0.54 & 0.63 & 0.35 & 0.62 & 0.46 & 0.33 & 0.71 & 0.29 \\
\hline Tebuconazole & 0.19 & 0.03 & 0.08 & 0.12 & 0.08 & 0.07 & 0.21 & 0.08 & 0.11 \\
\hline Fluazinam & 0.10 & 0.04 & 0.13 & 0.01 & 0.09 & 0.01 & 0.16 & 0.05 & 0.03 \\
\hline
\end{tabular}

${ }^{\mathrm{z}}$ Effective concentration for 50\% inhibition values. 
conditions, as well as four sensitive wild-type isolates, found that the resistant mutants had reduced fitness with regard to both pathogenicity and osmotic stress. Furthermore, the mutants exhibited higher levels of glycerol accumulation, all of which are consistent with the findings of other studies investigating the ascomycete pathogen B. cinerea (Gong et al. 2018; Ren et al. 2016; Zhou et al., 2020). Similar findings have also been reported in studies of $F$. asiaticum in China (Qiu et al. 2018). It is also interesting to note that dimethachlone-resistant isolates of Sclerotinia sclerotiorum and fenhexamid-resistant mutants of $B$. cinerea also exhibit similar patterns of fitness and virulence (Zhou et al. 2014, 2017).

Although fludioxonil has strong activity against a wide range of plant pathogens, and has extensively been used for the control of numerous diseases over the last 30 years, the mode of action of fludioxonil has still not been characterized in detail (Brandhorst et al. 2019; Koch and Leadbeater 1992; Qiu et al. 2018; Sang et al. 2018). However, many studies have indicated that the protein target of fludioxonil is an HK associated with the HOG1 cascade MAP kinase signaling pathway and that fludioxonil-resistant mutants have increased sensitivity to osmotic stress (Brandhorst et al. 2019; Gong et al. 2018; Qiu et al. 2018; Ren et al. 2016). The latter observation is of great interest because it is well known that the fitness of resistant mutants is an extremely important parameter regarding the risk for development of fungicide resistance (Brent and Hollomon 2007). Cross resistance between different fungicides is also an important risk factor and, although there were insufficient studies to determine the full extent of cross resistance between fludioxonil and other fungicides, the results from investigations focusing on $S$. homoeocarpa and B. cinerea have indicated that fludioxonil resistance can lead to reduced sensitivity to dicarboximide fungicides such as iprodione, vinclozolin, and tolclofos-methyl but not to other fungicides such as propiconazole, fenpropimorph, cyprodinil, tridemorph, epoxiconazole, spiroxamine, fluazinam, and chlorothalonil. Conversely, a study of fludioxonilresistant mutants of Aspergillus carbonarius actually found an increased sensitivity to the strobilurin pyraclostrobin (Malandrakis et al. 2013). The results of the current study reconfirmed these prior observations, finding a positive cross resistance between fludioxonil and the dicarboximide fungicides procymidone and iprodione but none with any of the other fungicides tested, including boscalid, carbendazim, tebuconazole, and fluazinam (Table 2). These results are not particularly surprising because it is known that fludioxonil and dicarboximides share similar mode of action. Taken together, these results indicated that the inclusion of fludioxonil in conjunction with nondicarboximide fungicides within an integrated pest management (IPM) program could help to minimize the risk of fludioxonil resistance development.

Several reports have suggested that mutations in the Os-1 HK upstream of the HOG1 MAP kinase signaling pathway can contribute to fludioxonil resistance in Alternaria brassicicola and B. cinerea, especially mutations in the $\mathrm{N}$-terminal region of the $O s 1$ gene (Avenot and Simoneau 2005; Ren et al. 2016). Similarly, several amino acid mutations in the FgOs1 of $F$. asiaticum have been linked to fludioxonil resistance, as well as changes in its $\mathrm{FgOs} 4$ and $\mathrm{FgOs} 5$ genes (Qiu et al. 2018). The molecular analysis conducted in the current study found evidence of similar mutations in $F$. graminearum. For example, the FgOsl gene of the fludioxonil-resistant mutant HBXT2R contained two point mutations that resulted in amino acid changes at $\mathrm{K} 223 \mathrm{~T}$ and $\mathrm{K} 415 \mathrm{R}$ in addition to a premature stop codon in its $\mathrm{FgOs} 5$ gene, while the resistant mutants 2XZ-4R, CM2R, and SQ1-2R had single point mutations in their $\mathrm{FgOs} 5$ genes that resulted in changes at K192R, K293R, and K411R, respectively. These point mutations were different from the previous reports (Supplementary Table S4) of the fludioxonil resistance in the MAP kinase protein in $F$. graminearum and $F$. asiaticum. However, none of the resistant mutants were found to have mutations in their $\mathrm{FgOs} 2$ and $\mathrm{FgOs} 4$ genes, and it is interesting to note that none of the mutations found in the $\mathrm{FgOs} \mathrm{I}$ and $\mathrm{FgOs} 5$ genes were the same as those documented in the F. asiaticum study (Qiu et al. 2018). However, the FgOs2 and $\mathrm{FgOs} 4$ expression of all of the fludioxonil-resistant mutants was significantly $(P<0.05)$ downregulated compared with the sensitive isolates (except for the SQ1-2 isolate), indicating that the candidate target genes $\mathrm{FgOs} 2$ and $\mathrm{FgOs} 4$ may also be related to the fludioxonil resistance, which was similar to dimethachlone resistance to $S$. sclerotiorum ( $\mathrm{Li}$ et al. 2017). It is also noteworthy that the fludioxonilresistant mutant YN1-3R did not have mutations in any of its candidate target genes. Taken together, these findings indicated that the mechanism of fludioxonil resistance in $F$. graminearum could be much more complex than previously thought, and could involve alternative resistance mechanisms. Further molecular-genetic analysis is required to completely characterize the mechanism of fludioxonil resistance in this species.

In summary, the current study found that no evidence of field resistance to fludioxonil in any of the $F$. graminearum samples collected from the six primary wheat-producing provinces of China. Analysis of the biological characteristics of five laboratory-induced fludioxonil-resistant mutants found significantly reduced fitness with regard to both osmotic sensitivity and pathogenicity. Considering the

Table 3. Mutations in the $\mathrm{FgOs} 1, \mathrm{FgOs} 2, \mathrm{FgOs} 4$, and $\mathrm{FgOs} 5$ protein sequence of fludioxonil-resistant mutants of Fusarium graminearum ${ }^{\mathrm{y}}$

\begin{tabular}{|c|c|c|c|c|}
\hline \multicolumn{3}{|c|}{ Location } & \multirow[b]{2}{*}{ Mutants } & \multirow[b]{2}{*}{ Mutation type } \\
\hline Genes & Types $^{\mathbf{z}}$ & Gene numbers & & \\
\hline $\mathrm{FgOs} 1$ & Histidine kinase & FGSG_07118 & HBXT2R & K223T, K415R \\
\hline $\mathrm{FgOs} 5$ & MAPKK & FGSG_08691 & $2 \mathrm{XZ}-4 \mathrm{R}$ & K192R \\
\hline $\mathrm{FgOs} 5$ & ND & FGSG_08691 & HBXT2R & 520 stop \\
\hline $\mathrm{FgOs} 5$ & ND & FGSG_08691 & CM2R & K293R \\
\hline $\mathrm{FgOs} 5$ & ND & FGSG_08691 & SQ1-2R & K411R \\
\hline $\mathrm{FgOs} 2$ & MAPK & FGSG_09612 & ND & ND \\
\hline $\mathrm{FgOs} 4$ & MAPKKK & FGSG_00408 & ND & ND \\
\hline
\end{tabular}

${ }^{y} \mathrm{ND}$ indicates no data available.

${ }^{\mathrm{z}}$ MAPKK $=$ mitogen-activated protein $(\mathrm{MAP})$ kinase kinase, $\mathrm{MAPK}=$ MAP kinase, and MAPKKK = MAP kinase kinase kinase.

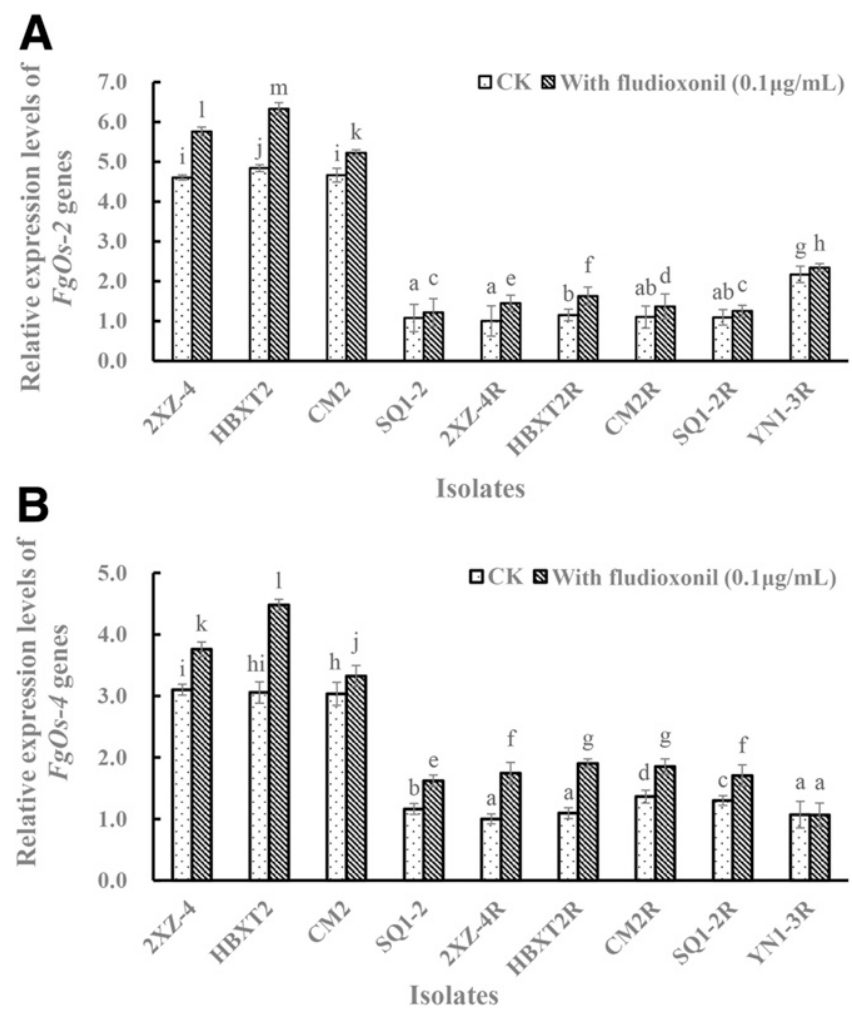

Fig. 5. Expression levels of $\mathbf{A}, \mathrm{FgOs} 2$ and $\mathbf{B}, \mathrm{FgOs} 4$ genes in fludioxonil-resistant mutants and sensitive isolates in Fusarium graminearum. $\mathrm{CK}=$ control. Values are means ( \pm standard error) of two independent repeats. Error bars denote the standard error of the mean according to Fisher's least significant difference test $(\alpha=0.05)$. 
critical role of chemical control in the management of FHB, it is important that measures are taken to manage the risk of fungicide resistance development. The observation that the fludioxonil-resistant mutants assessed in the current study exhibited no cross resistance with boscalid, carbendazim, tebuconazole, and fluazinam indicates that the combined or alternated application of fludioxonil with these fungicides within an IPM program could provide effective and sustained control of FHB in wheat production. However, despite these precautions, the discovery of the YN1-3R mutant, which appears to have a novel mechanism of resistance, demonstrates that it is important to continue monitoring fludioxonil resistance in the field, and that further research is still required to completely characterize the mechanisms of resistance in $F$. graminearum and guarantee the future safety of wheat production in China.

\section{Literature Cited}

Al-Taweel, K., Dilantha Fernando, W. G., and Brule-Babel Anita, L. 2014. Transcriptome profiling of wheat differentially expressed genes exposed to different chemotypes of Fusarium graminearum. Theor. Appl. Genet. 127: 1703-1718.

Avenot, H., Simoneau, P., Iacomi-Vasilescu, B., and Bataillé-Simoneau, N. 2005. Characterization of mutations in the two-component histidine kinase gene AbNIK1 from Alternaria brassicicola that confer high dicarboximide and phenylpyrrole resistance. Curr. Genet. 47:234-243.

Avenot, H. F., and Michailides, T. J. 2015. Detection of isolates of Alternaria alternata with multiple resistance to fludioxonil, cyprodinil, boscalid and pyraclostrobin in California pistachio orchards. Crop Prot. 78:214-221.

Bailey, D. J., Paveley, N., Pillinger, C., Foulkes, J., Spink, J., and Gilligan, C. A. 2005. Epidemiology and chemical control of take-all on seminal and adventitious roots of wheat. Phytopathology 95:62-68.

Blandino, M., Haidukowski, M., Pascale, M., Plizzari, L., Scudellari, D., and Reyneri, A. 2012. Integrated strategies for the control of Fusarium head blight and deoxynivalenol contamination in winter wheat. Field Crops Res. 133:139-149.

Brandhorst, T. T., Kean, I. R. L., Lawry, S. M., Wiesner, D. L., and Klein, B. S. 2019. Phenylpyrrole fungicides act on triosephosphate isomerase to induce methylglyoxal stress and alter hybrid histidine kinase activity. Sci. Rep. 9: 5047.

Brent, K. J., and Hollomon, D. W. 2007. Fungicide Resistance: The Assessment of Risk, 2nd revised ed. FRAC Monogr. 2. Fungicide Resistance Action Committee, CropLife International, Brussels, Belgium.

Brent, K. J., and Hollomon, D. W. 1998. Pages 1-48 in: Fungicide Resistance: The Assessment of Risk. Global Crop Protection Federation, Brussels, Belgium.

Broders, K. D., Lipps, P. E., Paul, P. A., and Dorrance, A. E. 2007. Evaluation of Fusarium graminearum associated with corn and soybean seed and seedling disease in Ohio. Plant Dis. 91:1155-1160.

Catlett, N. L., Yoder, O. C., and Turgeon, B. G. 2003. Whole-genome analysis of two-component signal transduction genes in fungal pathogens. Eukaryot. Cell 2:1151-1161.

Chen, Y., Wang, W. X., Zhang, A. F., Gu, C. Y., Zhou, M. G., and Gao, T. C. 2011. Activity of the fungicide JS399-19 against Fusarium head blight of wheat and the risk of resistance. J. Integr. Agric. 10:1906-1913.

Chilaka, C., De Boevre, M., Atanda, O., and Saeger, S. D. 2017. The status of Fusarium mycotoxins in Sub-Saharan Africa: A review of emerging trends and post-harvest mitigation strategies towards food control. Toxins (Basel) $9 \cdot 19$.

De Miccolis Angelini, R. M., Rotolo, C., Masiello, M., Gerin, D., Pollastro, S., and Faretra, F. 2014. Occurrence of fungicide resistance in populations o Botryotinia fuckeliana (Botrytis cinerea) on table grape and strawberry in south Italy. Pest Manage. Sci. 70:1785-1796.

Dry, I. B., Yuan, K. H., and Hutton, D. G. 2004. Dicarboximide resistance in field isolates of Alternaria alternata is mediated by a mutation in a two-component histidine kinase gene. Fungal Genet. Biol. 41:102-108

Duan, Y. B., Ge, C. Y., and Zhou, M. G. 2014a. Molecular and biochemical characterization of Sclerotinia sclerotiorum laboratory mutants resistant to dicarboximide and phenylpyrrole fungicides. J. Pest Sci. 87:221-230.

Duan, Y. B., Li, M. X., Zhao, H. H., Lu, F., Wang, J. X., and Zhou, M. G. 2018. Molecular and biological characteristics of laboratory metconazole-resistant mutants in Fusarium graminearum. Pestic. Biochem. Physiol. 152:55-61.

Duan, Y. B., Zhang, X. K., Ge, C. Y., Wang, Y., Cao, J. H., Jia, X. J., Wang, J. X., and Zhou, M. G. 2014b. Development and application of loop-mediated isothermal amplification for detection of the F167Y mutation of carbendazim-resistant isolates in Fusarium graminearum. Sci. Rep. 4:7094.

Fillinger, S., Ajouz, S., Nicot, P. C., Leroux, P., and Bardin, M. 2012. Functional and structural comparison of pyrrolnitrin and iprodione-induced modifications in the class III histidine-kinase Bos1 of Botrytis cinerea. PLoS One 7:e42520.

Furukawa, K., Randhawa, A., Kaur, H., Mondal, A. K., and Hohmann, S. 2012. Fungal fludioxonil sensitivity is diminished by a constitutively active form of the group III histidine kinase. FEBS Lett. 586:2417-2422.
Gong, C. W., Qin, Y. M., Qu, J. S., and Wang, X. G. 2018. Resistance detection and mechanism of strawberry Botrytis cinerea to fludioxonil in Sichuan Province. Sci. Agric. Sin. 51:4277-4287.

Goswami, R. S., and Kistler, H. C. 2004. Heading for disaster: Fusarium graminearum on cereal crops. Mol. Plant Pathol. 5:515-525.

Haile, J. K., Diaye, A., Walkowiak, S., Nilsen, K. T., Clarke, J. M., Kutcher, H. R. Steiner, B., Buerstmayr, H., and Pozniak, C. J. 2019. Fusarium Head Blight in durum wheat: Recent status, breeding directions, and future research prospects. Phytopathology 109:1664-1675.

Han, X., Zhao, H., Ren, W. C., Lv, C. Y., and Chen, C. J. 2017. Resistance risk assessment for fludioxonil in Bipolaris maydis. Pestic. Biochem. Physiol. 139:32-39.

Hu, J., Zhou, Y. X., Gao, T., Geng, J. M., Dai, Y., Ren, H. Y., Lamour, K., and Liu, X. L. 2019. Resistance risk assessment for fludioxonil in Sclerotinia homoeocarpa in China. Pestic. Biochem. Physiol. 156:123-128.

Jung, K., Fried, L., Behr, S., and Heermann, R. 2012. Histidine kinases and response regulators in networks. Curr. Opin. Microbiol. 15:118-124.

Koch, E., and Leadbeater, A. J. 1992. Phenylpyrroles-A new class of fungicides for seed treatment. Pages 1137-1146 in: Brighton Crop Prot. Conf. Pests Dis. British Crop Protection Council.

Lew, R. R. 2010. Turgor and net ion flux responses to activation of the osmotic MAP kinase cascade by fludioxonil in the filamentous fungus Neurospora crassa. Fungal Genet. Biol. 47:721-726.

Li, H. X., and Xiao, C. L. 2008. Characterization of fludioxonil-resistant and pyrimethanil-resistant phenotypes of Penicillium expansum from apple. Phytopathol. 98:427-435.

Li, J. L., Kang, T. H., Talab, K. M. A., Zhu, F. X., and Li, J. H. 2017. Molecular and biochemical characterization of dimethachlone resistant isolates of Sclerotinia sclerotiorum. Pestic. Biochem. Physiol. 138:15-21.

Liu, S. M., Duan, Y. B., Ge, C. Y., Chen, C. J., and Zhou, M. G. 2013. Functional analysis of the $\beta 2$-tubulin gene of Fusarium graminearum and the $\beta$-tubulin gene of Botrytis cinerea by homologous replacement. Pest Manage. Sci. 69: 582-588.

Liu, S. M., Fu, L. Y., Wang, S., Chen, J. P., Jiang, J., Che, Z. P., Tian, Y., and Cheng, G. Q. 2019. Carbendazim resistance of Fusarium graminearum from Henan wheat. Plant Dis. 103:2536-2540.

Malandrakis, A. A., Vattis, K. N., Doukas, E. G., and Markoglou, A. N. 2013 Effect of phenylpyrrole resistance on fitness parameters and ochratoxin production in Aspergillus carbonarius. Int. J. Food Microbiol. 165: 287-294.

Miller, T. K., Renault, S., and Selitrennikoff, C. P. 2002. Molecular dissection of alleles of the osmotic-1 locus of Neurospora crassa. Fungal Genet. Biol. 35: 147-155.

Peters, R. D., Platt, H. W., Drake, K. A., Coffin, R. H., Moorehead, S., Clark, M. M., Al-Mughrabi, K. I., and Howard, R. J. 2008. First report of fludioxonil-resistant isolates of Fusarium spp. causing potato seed-piece decay. Plant Dis. 92:172.

Qian, H. W., Du, J., Chi, M. Y., Sun, X. M., Liang, W. X., Huang, J. G., and Li, B. D. 2018. The Y137H mutation in the cytochrome P450 FgCYP51B protein confers reduced sensitivity to tebuconazole in Fusarium graminearum. Pest Manage. Sci. 74:1472-1477.

Qiu, J., and Shi, J. 2014. Genetic relationships, carbendazim sensitivity and mycotoxin production of the Fusarium graminearum populations from maize, wheat and rice in eastern China. Toxins (Basel) 6:2291-2309.

Qiu, J. B., Yu, M. Z., Yin, Q., Xu, J. H., and Shi, J. R. 2018. Molecular and characterization, fitness and mycotoxin production of Fusarium asiaticum strains resistant to fludioxonil. Plant Dis. 102:1759-1765.

Rawat, N., Pumphrey, M. O., Liu, S. X., Zhang, X. F., Tiwari, V. K., Ando, K., Trick, H. N., Bockus, W. W., Akhunov, E., Anderson, J. A., and Gill, B. S. 2016. Wheat Fhb1 encodes a chimeric lectin with agglutinin domains and a pore-forming toxin-like domain conferring resistance to Fusarium head blight. Nat. Genet. 48:1576-1580.

Ren, W. C., Shao, W. Y., Han, X., Zhou, M. G., and Chen, C. J. 2016. Molecular and biochemical characterization of laboratory and field mutants of Botrytis cinerea resistant to fludioxonil. Plant Dis. 100:1414-1423.

Sang, C. W., Ren, W. C., Wang, J. J., Xu, H., Zhang, Z. H., Zhou, M. G., Chen, C. J., and Wang, K. 2018. Detection and fitness comparison of target-based highly fludioxonil-resistant isolates of Botrytis cinerea from strawberry and cucumber in China. Pestic. Biochem. Physiol. 147:110-118.

Tateishi, H., Miyake, T., Moi, M., Kimura, R., Sakuma, Y., and Saishoji, T. 2010. Sensitivity of Japanese Fusarium graminearum species complex isolates to metconazole. J. Pestic. Sci. 35:419-430.

Tückmantel, S., Greul, J. N., Janning, P., Brockmeyer, A., Grütter, C., Simard, J. R., Gutbrod, O., Beck, M. E., Tietjen, K., Rauh, D., and Schreier, P. H 2011. Identification of Ustilago maydis aurora kinase as a novel antifungal target. ACS Chem. Biol. 6:926-933.

Willyerd, K. T., Li, C., Madden, L. V., Bradley, C. A., Bergstrom, G. C., Sweets, L. E., McMullen, M., Ransom, J. K., Grybauskas, A., Osborne, L., Wegulo, S. N., Hershman, D. E., Wise, K., Bockus, W. W., Groth, D., Dill-Macky, R., Milus, E. E., Sker, P. D., Waxman, K. D., Adee, E. A., Ebelhar, S. E., Young, B. G., and Paul, P. A. 2012. Efficacy and stability of integrating fungicide and cultivar resistance to manage Fusarium head blight and deoxynivalenol in wheat. Plant Dis. 96:957-967. 
Wu, D. X., Zhang, R. S., Han, X., Wang, J. X., Zhou, M. G., and Chen, C. J. 2015. Resistance risk assessment for fludioxonil in Stemphylium solani. Ann. Appl. Biol. 167:277-284

Yang, Y., Li, M. X., Duan, Y. B., Li, T., Shi, Y. Y., Zhao, D. L., Zhou, Z. H., Xin, W. J., Wu, J., Pan, X. Y., Li, Y. J., Zhu, Y. Y., and Zhou, M. G. 2018. A new point mutation in $\beta 2$-tubulin confers resistance to carbendazim in Fusarium asiaticum. Pestic. Biochem. Physiol. 145:15-21.

Yoshimi, A., Tsuda, M., and Tanaka, C. 2004. Cloning and characterization of the histidine kinase gene Dic1 from Cochliobolus heterostrophus that confers dicarboximide resistance and osmotic adaptation. Mol. Genet. Genomics 271: 228-236.

Yuan, S., and Zhou, M. G. 2005. A major gene for resistance to carbendazim, in field isolates of Gibberella zeae. Can. J. Plant Pathol. 27:58-63.

Zhang, Y., Chen, W., Shao, W., Wang, J., Lv, C., Ma, H., and Chen, C. 2017. Molecular, biological and physiological characterizations of resistance to phenamacril in Fusarium graminearum. Plant Pathol. 66: 1404-1412.
Zhang, Y.-J., Yu, J.-J., Zhang, Y.-N., Zhang, X., Cheng, C.-J., Wang, J.-X., Hollomon, D. W., Fan, P.-S., and Zhou, M.-G. 2009. Effect of carbendazim resistance on trichothecene production and aggressiveness of Fusarium graminearum. Mol. Plant-Microbe Interact. 22:1143-1150.

Zhang, Y.-J., Zhang, X., Chen, C.-J., Zhou, M.-G., and Wang, H.-C. 2010. Effects of fungicides JS399-19, azoxystrobin, tebuconazole, and carbendazim on the physiological and biochemical indices and grain yield of winter wheat. Pestic. Biochem. Physiol. 98:151-157.

Zhou, F., Hu, H. Y., Song, Y. L., Gao, Y. Q., Liu, Q. L., Song, P. W., Chen, E. Y., Yu, Y. A., Li, D. X., and Li, C. W. 2020. Biological characteristics and molecular mechanism of fludioxonil resistance in Botrytis cinerea from Henan Province of China. Plant Dis. 104:1041-1047.

Zhou, F., Lu, F., Zhang, C., Qi, H. X., Wang, X. D., and Zhang, G. Z. 2017. Occurrence of fenhexamid resistance in Botrytis cinerea from greenhouse strawberries in China. J. Phytopathol. 165:455-462.

Zhou, F., Zhang, X. L., Li, J. L., and Zhu, F. X. 2014. Dimethachlon resistance in Sclerotinia sclerotiorum in China. Plant Dis. 98:1221-1226. 